\title{
AN EXPERIMENT ON THE USE OF PSYCHOLOGICAL TESTS AS AN AID TO THE REHABILITATION OF HOSPITAL PATIENTS
}

\author{
BY \\ ALICE W. HEIM and AUDREY RENNISON \\ From the Applied Psychology Research Unit (Medical Research Council), Cambridge
}

\section{Introduction}

The following report is based on data which have been collected at the Birmingham Accident Hospital and Rehabilitation Centre between January and November, 1945. The work was initiated as a result of discussions between the hospital's assistant almoner and two members of the Medical Research Council's Applied Psychology Unit. These discussions centred on the applicability of vocational tests, which have been designed for subjects of normal physique, to patients attending the hospital for treatment and rehabilitation.

The Birmingham Accident Hospital treats patients who have had injuries from any cause, but the industrial accident predominates, and the emphasis in treatment is to fit the patient once more for his full work. The patient is approached as an individual and his injury related to his social background and his work. His treatment may include class work and games in the gymnasium, and work in the Occupational Therapy Department. It requires his active co-operation, and holds his interest. Most patients attend daily, and there are few absentees. Most patients, when treatment is complete, return to their old occupations, but a few-because of a residual disability, or because they were seriously ill-adjusted to their former occupation-need a change of work.

It is clear that the suitability of their choice of occupation plays an important part in the mental and physical rehabilitation of these patients. It was decided, therefore, to try out a small battery of psycholcgical tests on patients whose future work was uncertain. Such patients, it was found, tended to fall into one of the two following classes: $(a)$ those who had no ideas on the subject, (b) those who wanted to enter an occupation judged by the hospital authorities to be quite unsuitable.

The tests used were as follows:

1. A.H.4, a pencil and paper intelligence test in two parts. Both parts aim at testing general mental capacity and not specific aptitudes, but Part I has a verbal and arithmetical bias and Part II has a visual bias. The qualities tested are: ability to reason, to obey simple instructions, to understand the meaning of everyday words and to observe details accurately.

The subject is asked to work through examples of the test questions in his own time in order to make sure that he understands his task and to help to dissipate any nervousness induced by the test situation. Ten minutes are allowed for each part of the test proper.

2. Mechanical Ability. This is a performance test consisting of five mechanical models which are partly taken to pieces. The subject is asked to fit up as many of these models as he can in 15 minutes. $\mathrm{He}$ is given a screw-driver, and is told that each model is separate and complete and that the parts which are already fitted should not be dismantled. He is not informed what function the models should fulfil when they have been correctly assembled.

3. National Institute of Industrial Psychology's Form Relations Test. This is a paper and pencil test of spatial perception. The subject is required to apprehend the shapes and sizes of different figures and the relations between them.

\section{Manual Dexterity}

(a) Screw Plate.

(b) Rings.

In the screw plate test, the subject is given 2 minutes in which to insert as many brass screws as possible through holes drilled in the top of a screw plate. There are 29 holes in all, and inside the apparatus is a steel tube which has holes tapped in it exactly opposite the holes in the top. The subject is asked to take the holes in order, working from right to left, and to screw each screw down as far as it will go, into the inner hole. (There is a stop which prevents the subject from giving more than three or four turns, in order to avoid testing conscientiousness in addition to dexterity.)

The dexterity consists in picking up the screws, one at a time, from the standard bowl; in placing them through the top hole at a right angle to ensure that they enter the inner hole and do not fall down the side of the tube; and in giving the requisite three turns, quickly and neatly. One hand only is used for handling the screws; the other may be used to steady the screw plate. 
The apparatus for the rings test consists of three key rings - one large, one medium and one smalland 35 curtain rings of slightly smaller diameter than that of the medium key ring. The subject is given the large key ring first and is asked to put on to it as many curtain rings as possible in 2 minutes. The curtain rings have to be picked up and attached one at a time, and each ring must be completely on before the next is attempted. After a short rest, the subject goes through the same procedure with the medium key ring and finally with the small one.

The score on the large key ring is ignored as its purpose is to act as a 'shock absorber' and to enable the subject to develop a technique. The sum of the scores gained on the medium and small rings is taken as the test score. Both hands are used in this test and each subject evolves his own technique, some using the curtain ring as a lever to open the key ring, some using their finger-nails, etc.

5. Alignment. The apparatus for this test consists of twenty brass rods and a box with a moveable cover. Four rows of five holes are drilled in the front plate of the box. Inside it, is another plate with a great many holes in it, including twenty which are exactly opposite the holes in the front. The distance between the two plates is slightly shorter than the length of the rods.

The subject is told that the inner plate has holes in it exactly opposite the holes in the outer plate, but he is not told of the greater number of holes in the inner plate. He is asked to pick up the rods one at a time and, beginning with the top row, to work across from left to right, placing each rod very straight through the outer hole, so that it goes into its inner hole, exactly opposite. He does this for two separate minutes, his score being the sum of the scores obtained in the 2 minutes.

Each rod correctly placed scores 3 marks; each rod slightly out in one plane scores 2 marks; each rod slightly out in both planes scores 1 mark. A rod which is placed in a hole in the inner plate, which is considerably out in one or both planes, scores 0 . This test is more experimental than the other tests which were used. Its aim is to assess the subject's sense of alignment-his capacity to guide the end of a tool which he cannot see.

Not all the subjects took all these tests. In some cases of hand injury, for instance, the rings test would have been impossible. However, the tester encouraged her subjects to try as many tests as possible, believing that in some cases an objective test might detect abilities which would surprise the hospital authorities and the patient-perhaps instructing the former and encouraging the latter.

Testing procedure was, inevitably, less uniform than is permissible with groups of more normal subjects. In certain cases, for example, with the mechanical ability test, the subject had to tell the tester how to assemble the models as, owing to a hand injury, he was unable to put his own ideas into practice.
Twenty-one patients were tested in all. Testing was done by the assistant almoner, who had previously received a short course of training in giving, marking and interpreting tests at the Cambridge Psychological Laboratory. Close contact was maintained between her and the interested members of the Medical Research Council's Applied Psychology Unit throughout the investigation-which latter terminated on the tester's leaving the Birmingham Accident Hospital in November 1945.

The following report has been written by the assistant almoner who had the performance test models made for the hospital and carried out all the testing and following-up. The Appendix consists of six illustrative case histories, chosen from the collection of twenty-one.

\section{Test Reports}

The reports appended are copies of those written on the case papers of the patients concerned, and are probably less interesting in this form than in their proper context. More information was available to the writer in the form of full social casehistories and medical notes. Relevant information, particularly about follow-up, is given in the reports.

In two cases (cases 2 and 5) test results were used as a guide to further treatment and were recommended for this purpose in the second case by the surgeon, and in the first, the occupational therapist concerned. This idea seems worth developing in a rehabilitation centre where results depend very much on a patient's ability to co-operate intelligently in treatment.

In two cases (cases 2 and 6) test results shed a new light on the patient's character:

Case 2 was believed to be idle and careless. She was well-spoken and chatted in a lively way, and was believed to be intelligent. Test results suggested that this was not so, and it was thought that she would benefit by treatment requiring less mental effort. When this adjustment was made, her progress justified the change.

Case 6 was thought to be unintelligent and completely lacking in any particular talent or interest. Test results showed that he was quite intelligent, and appeared to have some ability on the mechanical side: Resettlement is now being followed on these lines, and the patient is working part-time with a firm of instrument makers while continuing treatment half the day with us.

The writer believed that case 6 was slightly ' shellshocked' and thought that an interest in work or plans for work would help him. He has recently shown spontaneous interest and pleasure in discussing his new job, though so far in nothing else.

Case 5 insisted on taking a mechanics job against advice based on test results. He was unable to do the work, and found that he disliked it. He is now training as a gardener as he particularly wanted out-door work, and it is hoped that this will prove more satisfactory. 
A patient J. N. was recommended clerical work, as he had a high leg amputation and needed work of a sedentary nature. He insisted that he disliked the idea, and was no good at clerical work, having had plenty of opportunity to find out for himself in the army. He was believed to be a capable man. He was not pressed further until test results were available, when it was found that clerical work was probably not his best line, and he was very happily resettled on instrument assembly.

This patient also found a car for himself, and altered the controls for one leg, without help or advice, so that his disability did not prevent him from driving.

In the majority of cases the tests were given to patients about whom a great deal was already known, so that information obtained in this way was largely confirmatory. In recent cases, chiefly with ex-service patients, this was not so, and it was interesting to see test results borne out in treatment.

The plan for ex-service patients referred for the last stage of their treatment was as follows: Treatment usually involved daily attendance in the Physiotherapy Department, the Occupational Therapy Department, and Gymnasium, and finally resettlement into suitable work. Patients were to be seen on the first attendance or as soon as possible afterwards, and problems of treatment and resettlement discussed. If resettlement was likely to be needed later, patients were to be given tests as soon as they had settled into the routine and atmosphere of the hospital. So far only cases 5, 6 and R. K., have come for testing under this scheme, and unfortunately just when the numbers of ex-service patients is increasing every week, the full scheme will have to be dropped.

\section{General Report on giving the Tests}

Every possible set-back in the form of convoys of service patients, staff shortage and ill-health has been met, and it has only been since March, 1945, that the experiment has been working satisfactorily. No difficulty has been encountered in persuading patients to do the tests, though it may take up to 2 hours of their day, and in some cases mean losing more time from work than they would normally in the course of treatment. (Patients were usually tested in time 'given' by the Occupational Therapy Department.) Tests were explained as a normal part of the hospital treatment and the resettlement service, and they were accepted as such. The two main difficulties have been shortage of time and noise in the hospital. A whole afternoon has to be devoted to each patient, partly because more than one case rarely occurred at any time, and also because by taking patients singly it was possible to watch their reactions closely. It was found to be impossible to find a quiet room. Even when people were prevented from interrupting, and the telephone was not allowed to ring, the usual noises of the hospital seemed all-pervading. Patients did not seem to notice the noise or to be disturbed by it, though the tester was always afraid that they might be.

\section{Specific Tests. Form Relations}

This test was found most interesting, because it was apparently a test of endurance for a certain type of patient. Section 6 appeared to be the point at which some patients broke down and refused to go any further. Two patients who were temperamentally rather volatile burst into tears at this point. It was interesting to see the patients growing more and more disheartened by their inability to see the answers easily but making an effort to get answers, then, when confronted with Section 6, giving in altogether, and after this ceasing to try.

\section{Alignment Test}

The two highest scores here were obtained by cases 1 and 6 , the former a good shot with a rifle, the latter a gun sighter in the Light Anti-aircraft Regiment.

This test was always given in the middle of the series, as patients seemed to enjoy doing it, and liked to have it taken to pieces afterwards. It revived interest and made a good 'break' half-way through.

\section{The Manual Dexterity Tests. Screw Plate}

Here scores were consistently lower than they should have been on industrial grading, and they would have fitted better with a grading as follows:

$\begin{array}{cc}\text { Industrial } & \text { Rehabilitation Centre } \\ \text { AB: } 20-29 & \text { AB: } 15-20 \\ \text { C: } 15-19 & \text { C: } 11-14 \\ \text { DE: } 9-14 & \text { DE: } 7-10\end{array}$

The twenty cases tried in this grade were very well distributed, but too few to be statistically significant.

Reports obtained independently, from the Occupational Therapy Department, on every patient tested also agreed with the lower grading.

\section{Rings Test}

This test interested the writer from the point of view of the intelligence factor: did intelligence play an important part in determining test score?-had this been taken into account when using the test with normal groups?

The writer has not given the rings test often enough to assess the intelligence factor statistically, but she found that clinically patients do show signs of thinking about the test (or not thinking, according to the type of patient) in a way which is not apparent with the screw plate test. By questioning, patients, who have failed to get any rings on during the test, can be led to think of methods for themselves. It is doubtful that this knowledge would ever be enough to lift a patient out of one grade into another.

Patients with some types of finger injuries appear to be unable to handle this test at all, though they 
can make a good attempt at the screw plate test. This is also true of patients who can use the fingers of one hand only.

\section{Co-operation with Hospital Staff}

Most of the assessment of a patient's ability and behaviour was done by the Occupational Therapy Department staff, and occasionally, the Gymnasium staff, who co-operated excellently and offered constructive help. There was no discrepancy found between test results and the patients' achievements in these departments, except in the two cases where there were contributory mental disorders. The medical staff also co-operated with the new service, particularly the senior surgeons, who were more often concerned with the type of patient involved. Patients were referred by the surgeon treating the patient primarily for testing, for example case 5, J. B., B. E. and C. L. Co-operation of such a satisfactory nature might be less possible where the contact between medical staff and almoners was not so well established, or where rather more conservative hopsital routine might put a brake on a new method of this kind.

\section{Co-operation with Other Agencies}

Information based on test results and recommendations suggesting suitable work were given to Ministry of Labour Resettlement Officers, and were received without question, and usually acted upon. Industrial personnel were more interested in the idea and asked questions about the tests used. The following examples of outside co-operation are recorded:

\section{Ministry of Labour}

Patient J. N. The Disablement Rehabilitation Officer of a small Labour Exchange in the country waited until he had information about the patient's abilities before deciding what type of work to find for him, and acted upon the advice given.

\section{Industry}

The Labour Manager of a firm of Scientific Instrument Makers had recently been demobilized. He was previously a colonel in the Light Antiaircraft Regiment, and on being told the whole story of difficulties in case 6 and taking into account the test results, employed him as a part-time worker until he should be well enough to stop treatment. If he proved efficient he could continue with this work at this particular firm. The patient was by no means prepossessing, and it is doubtful whether he would have been offered the job had it not been for belief in his ability resulting from these tests, and the Labour Manager's willingness to accept them.

\section{Other Hospitals}

Case 4 had been suffering for 4 years with a hand injury severe enough to necessitate an amputation. During this time he had been unable to use the stump because of pain, and had not learned to use his left hand. On his test results it seemed worth recommending that he should be taught to write and to use his left hand, and he was sent up to the Occupational Therapy Department where he made excellent progress at first. Later it was discovered that there were psychological factors which could not be treated in this hospital, and the patient was referred to a psychiatrist. A full report was sent with him, including his test scores and report, and this information proved relevant and helpful.

\section{Summary}

Although few patients have been tested, in the writer's opinion the results have shown that this work has a place in a hospital, and that it is capable of development. The outlook of medical, auxiliary and lay staff in the hospital concerned is encouraging to experiment, and the development of work on new lines. Each patient is treated as an individual and his injury related to his social background and his work, so that the tests fitted in well with the normal plan for treatment, both in theory and practice. The organization of a service of this kind does not normally come into the duties of an Almoner's Department, and if the tests were not given by a trained psychologist, expert guidance would have to be available to supervise the work and interpret the results.

\section{Case Reports}

Case 1. D. D. aged 31.

Diagnosis. Severe fractures in several places of right femur. (Injured on Active Service.)

Left school at 14. Attended Church of England School.

Last job: Army-corporal.

Job before last: shop assistant.

\section{General approach to test}

Patient was quite co-operative though nervous. $\mathrm{He}$ showed no signs of distress until the end of the last test of the Form Relations group, which he was unable to do, and after this he seemed tired and not at his ease with the Mechanical Ability Test. (The Almoner wondered whether the patient was not well enough to do an hour and a half of work at this time.)

\section{Special comments}

A.H.4. Patient was better on the first part than the second, although he had no difficulty with the examples of either. He made a few mistakes but proportionately more on the second part than the first.

Manual Dexterity. He was very moderate on the Screw Plate, but good on the Rings and surprisingly good on the Alignment. Patient worked rapidly without faltering and practically every rod scored on both attempts.

Form Relations. Patient lost heart in the last test, and could not be pressed to finish the time.

Mechanical Ability. Obviously this was not the patient's strong point, and again he lost interest about three minutes before the end of the allotted time. (This might have been fatigue.) He was not pressed to finish.

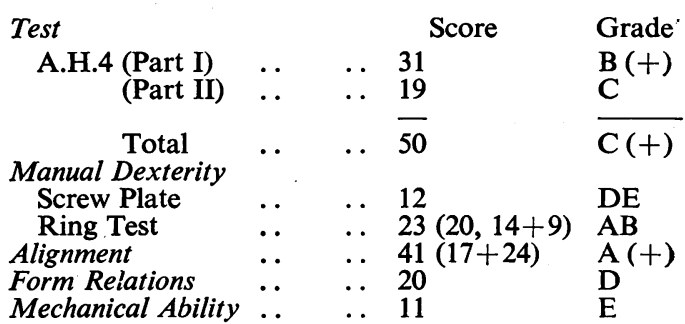



job.

Surgeon's Recommendations. He will require a sitting

\section{Conclusions}

Patient is quite capable and his shop-assistant's experience must have given him some opportunity to handle accounts and do clerical work. He would have to have a sitting job and he might not like full-time employment under factory conditions.

He has not a high mechanical ability score, although his manual dexterity is fairly good.

This patient might possibly do one of the lower grades of civil service job, or work as a ledger clerk or cashier. He might also be well suited to the work of telephone operator where his dexterity and his quick mental capacity would stand him in good stead.

Case 2. R. L., aged 39.

Diagnosis. Crushed right hand. Traumatic amputation of little finger.

Left school at 14 .

Last job: shell band machine.

Job before last: on scrap machine.

\section{Reason for test}

Patient was referred to Almoner because she was not progressing satisfactorily in the occupational therapy department. The Occupational Therapist could not decide whether she was lazy or unintelligent. Patient had similar symptoms to P.N. but this was not borne out by her test results. She seemed more stable in her approach to the tests and less intelligent.

\section{General approach to test}

Patient is a vivacious and talkative woman, who gives the impression of being much more able than she is. Her conversation and attitude to treatment suggest a certain degree of competence which her performance belies. Her general approach to test was confident and cheerful and although she was rather depressed by the number of careless mistakes she made, she did not think her performance was unsatisfactory. Her righthand injury seemed no disability.

\section{Special comments}

A.H.4. She worked through examples on her own but got nearly all of them wrong. This seemed partly carelessness and partly lack of understanding. Patient made several mistakes in the test proper, doing nearly as many questions wrong as right.

Manual Dexteritv. Patient was surprisingly good considering that her hand is badly deformed and still under operative treatment. Patient enjoyed doing the tests.

Alignment. Patient was careful but not very good. Made a good many bad shots.

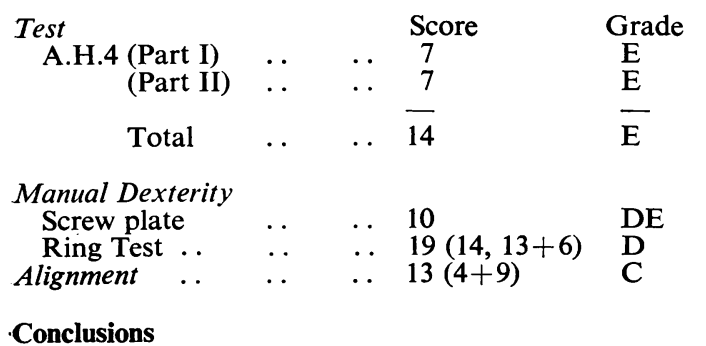

Patient seems capable of using her hands, provided the operation is not a difficult one, and her lively manner is no estimate of her mental ability.

\section{Follow-up}

23.10.45. In view of her long period of treatment, patient was given a holiday and then put back on simpler work, where progress was made.
Case 3. H. R., aged 31.

Diagnosis. Amputation above elbow of left arm.

Left school at 17. Attended Grammar School.

Last job: machine tool fitter and tool-room fitter.

Job before last: night entry clerk in bakery.

\section{General approach to test}

Patient was asked to do the test more from Almoner's interest and his own than for any specific resettlement problem. Patient was known to be intelligent and cooperative. He enjoyed doing the tests and had no difficulty in approaching them. Of the non-written tests only the Mechanical Ability was given, but patient was interested in the others and wanted to have them explained to him.

\section{Special comments}

A.H.4. He liked Part II, but did not do much better on it.

Form Relations. He did not like the last few tests but was not nervous when confronted with them. He wanted to sort out Test 8 at the end, and this was done.

Mechanical Ability. Almoner was not sure whether this should be given to patient because he might be embarrassed or discouraged by a test which should require the use of two hands. The results of A.H.4 and Form Relations suggested that it would be interesting to see patient's approach to the test as he seemed interested, and this was suggested. It was found that there was no difficulty in giving the test objectively and in.timing it as for a normal patient. The tester did help to the extent of holding a screw in place and putting the links of the chain together entirely on patient's instructions, which were perfectly clear and could not be misinterpreted. Patient never hesitated, he went straight through the tests and finished them with $8 \frac{1}{2}$ minutes to spare.

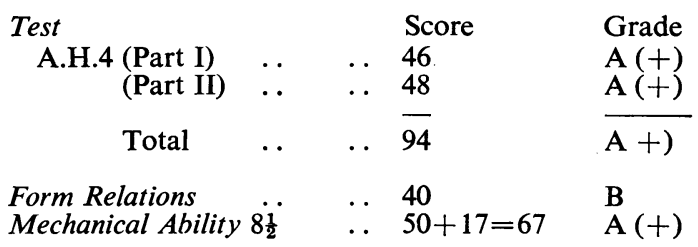

11.6.45. Patient may be found work at a plastics factory as a laboratory assistant. He is anxious to work on plastics and is going to do the City and Guild Examination as soon as he starts work.

20.6.45. Patient is to start work in the plastics laboratories on statistical control of experimental work. He is pleased with the prospect.

23.10.45. He is doing well at work. $\mathrm{He}$ is fitted with artificial limb.

Case 4. L. R., aged 50.

Diagnosis. Traumatic amputation all fingers right hand, reported here on 9.11.42.

Injury 2 years before-? Raynaud's disease

Left school at 13. Attended Council School.

Last job: stamp operator.

Job before last: engine driver in engineering works.

\section{General approach to test}

Patient surprisingly stolid and settled down very well, despite his very nervous manner and his nervous approach to treatment. He grasped instructions easily and listened carefully. He did not get flustered when faced with difficulties.

\section{Special comments}

A.H.4. He did the examples quickly in both parts and obtained an even score on each. He had no preferences. He had some difficulty because he could only write figures with his left hand, and this only slowly and in shaky writing, but it was felt that this would not 
have altered the grade of his score, which was consistent with his other results.

Form Relations. He started quite well but deteriorated at Section 6. He did not lose interest.

Manual Dexterity. As patient does not use his right hand and cannot write with his left hand it was impossible to give the Ring Test.

Screw Plate. He tackled this comfortably, without faltering, and although his score was not high, he seemed potentially capable of using his hand. He obtained a good result on his Alignment. (He develops a functional tremor of his left hand when using it consciously.)

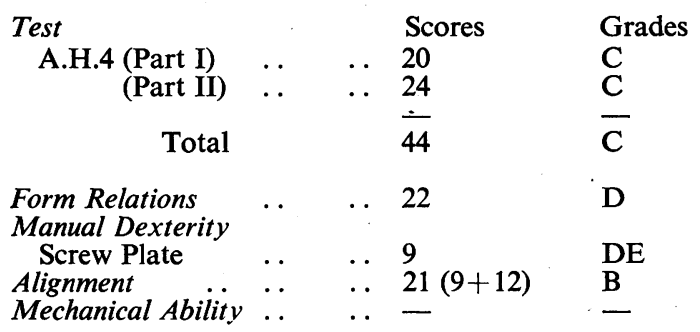

Special Recommendations. Refer to Occupational Therapy Department in order to teach this patient to use his left hand to write.

11.6.45. Patient made great strides in the Occupational Therapy Department and quickly learnt to write with his left hand. He wrote letters to his sons in the Army who had not heard from him personally for three or four years. As soon as the question of work was approached patient began to go downhill. He seemed disinclined to face the problem.

Finally, it was suggested that patient should obtain a job as cloak-room attendant and general attendant to the Gymnasium and Massage Departments. This job would not be heavy, but would entail responsibility, the care of money and people's personal belongings. Patient seemed inclined to consider this favourably.

30.7.45. The patient was no longer co-operating satisfactorily in treatment and his condition was diagnosed by a psychiatrist as an involution depression superimposed on a traumatic neurosis.

Case 5. F. T., aged 21.

Diagnosis. Ulnar nerve palsy of left hand (gun-shot wound).

Left school at $14 . \quad$ Attended Senior School.

Last job: private in infantry. (No trade.)

Job before last: labourer and guillotine saw operator.

\section{General approach to test}

Patient is a keen but not a reliable worker. $\mathrm{He}$ makes many careless mistakes, and does not think what he is about to do. His injury seems to be no disability as far as the Manual Dexterity Tests are concerned. The patient is illiterate. He can only just spell his own name and cannot read or make sense of a difficult sentence.

\section{Special comments}

A.H.4. He was very slow in Part I, but quicker in Part II. Patient was better at this part, although he took as long with the examples. Several of the longer words in the first test had to be substituted by simpler ones as patient was unable to read them or understand them.

Manual Dexterity. Patient was too impetuous and not careful. This showed particularly in the Alignment Test where he got nearly all the rods in and practically none of them right.

Mechanical Ability. Patient had no idea what he was doing with any of the models except the lock, on which he got the tongue in place and seemed to know vaguely what he was doing.

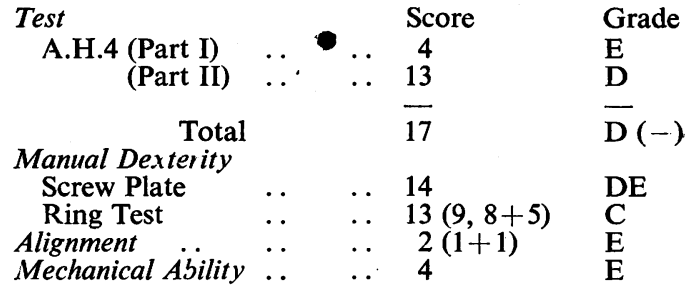

Comments. Patient's one ambition in life is to be a mechanic.

11.6.45. Patient found work as an unskilled mechanic in a transport garage. He says he likes the work very much. It is only a small firm but they take an interest in him and allow him to come for treatment. He has been seen at the Peripheral Nerve Injury Centre and his full disability pension is being continued.

30.7.45. Garage work was found to be unsatisfactory. Patient decided he did not like it. After some discussion he was recommended for training as a gardener, and is to go to a residential training centre for the course.

23.10.45. Now at training centre doing a gardening course.

Case 6. P. L., aged 28. 17.9.1945.

Diagnosis. Gun-shot wound left upper arm and left buttock. Has had a fracture of the left humerus. Muscular spiral palsy.

Last job: gunner, R.A.

Job before last: builder's labourer.

General Remarks. The patient was singularly disinterested in his future, but said he would like to do a course of carpentry and joinery. The almoner wondered whether he was suffering from 'shell shock.' He did not give the impression of ability or intelligence, but test results did not confirm this.

\section{General approach to test}

He tackled the tests methodically; and seemed to enjoy some of them. He had done army tests before.

\section{Special Comments}

A.H.4. He was slow on the first part, but surprisingly good on the second part and enjoyed it.

Manual Dexterity. He had the idea (which had been met before) that to open the key ring with the finger-nail would spoil the spring. (This was discovered after the test had been given.)

In view of the encouraging results on A.H.4 and the proposed joinery and carpentry training course, the Form Relations Test was given later.

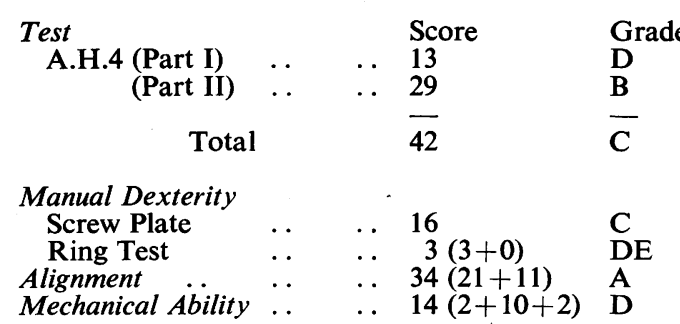

\section{Follow-up}

23.10.45. The patient was found temporary part-time work on instrument assembly until his arm was fit for the carpentry course; but character difficulties seemed likely to upset resettlement. As this man was one of the last patients tested, no further information was available at the time of writing the report. 